\title{
Riociguat therapy for pulmonary hypertension
}

$\mathrm{R}$ iociguat, a novel stimulator of soluble guanylate cyclase, improves walking distance and pulmonary vascular resistance in patients with either pulmonary arterial hypertension (PAH) or chronic thromboembolic pulmonary hypertension (CTEPH). These findings come from two phase III, international, randomized, controlled clinical trials: PATENT-1 and CHEST-1, respectively.

In PAH (classified by the WHO as group I pulmonary hypertension $[\mathrm{PH}])$, progressive vascular remodelling causes increased pulmonary vascular resistance, and right ventricular enlargement and failure. By contrast, CTEPH (WHO group IV PH) results from thromboembolic obstruction of the pulmonary vasculature, which also leads to increased pulmonary vascular resistance and right ventricular failure. $\mathrm{PH}$ owing to left heart disease is classified in WHO group II, whereas $\mathrm{PH}$ resulting from lung disease, hypoxia, or both, are assigned to WHO group III. WHO group V includes other miscellaneous causes of $\mathrm{PH}$, such as systemic and metabolic disorders.

Currently, PAH can be treated with phosphodiesterase type 5 (PDE5) inhibitors, prostanoids, or endothelinreceptor antagonists, but mortality remains high. Pulmonary endarterectomy is the standard treatment for CTEPH, but some patients are ineligible for surgery, and others have recurrent or persistent $\mathrm{PH}$ despite the procedure. No medical

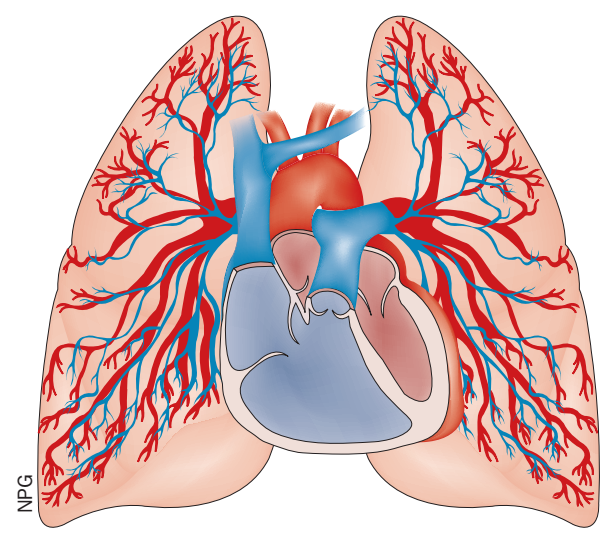

therapy for CTEPH is currently approved and, therefore, a considerable unmet need for efficacious therapy exists for both conditions.

Soluble guanylate cyclase stimulators are a novel class of agents that can produce vasodilatation via enhanced nitric oxidecyclic GMP signalling in the absence of endogenous nitric oxide. Notably, a limitation of PDE5 inhibitors is that they rely on the bioavailability of nitric oxide, which can be reduced in $\mathrm{PH}$.

In PATENT-I, 443 patients with symptomatic PAH (61\% idiopathic PAH, 25\% PAH associated with connective-tissue disease) were randomly allocated in a 2:4:1 ratio to receive a placebo, riociguat up to $2.5 \mathrm{mg}$ three times daily, or riociguat up to $1.5 \mathrm{mg}$ three times daily. At baseline, patients were receiving either no treatment for $\mathrm{PAH}$ (50\%), endothelin-receptor antagonists (44\%), or prostanoids (6\%). Patients taking PDE5 inhibitors were excluded. The primary analysis was between the first two groups; the last group was included for exploratory purposes only.

After 12 weeks, the mean 6 min walking distance had increased by $30 \mathrm{~m}$ in the $2.5 \mathrm{mg}$ riociguat group, and had decreased by $6 \mathrm{~m}$ in the placebo group $(P<0.001)$. This improvement occurred both in patients who were drug-naive at baseline, and in those taking endothelin-receptor antagonists or prostanoids. Patients who received riociguat also showed significant improvement in pulmonary vascular resistance, cardiac output, N-terminal pro-B-type natriuretic peptide level, time to clinical worsening, and WHO functional class.

In CHEST-1, 261 patients with inoperable CTEPH or persistent or recurrent $\mathrm{PH}$ after pulmonary endarterectomy were randomly assigned in a 1:2 ratio to receive a placebo or riociguat (final dose $0.5-2.5 \mathrm{mg}$ three times daily). Patients were excluded if they had received medical therapy for $\mathrm{PAH}$ in the previous 3 months.

At the 16-week follow-up, the mean 6 min walking distance had increased by $39 \mathrm{~m}$ in the riociguat group, and had decreased by $6 \mathrm{~m}$ in the placebo group $(P<0.001)$. Riociguat was also associated with improvements in pulmonary vascular resistance, cardiac output, $\mathrm{N}$-terminal pro-B-type natriuretic peptide level, and WHO functional class.

In an editorial that accompanied the trial publications in the New England Journal of Medicine, Stephen L. Archer heralds riociguat as a "safe and ... promising addition to the pharmacopeia for group I $\mathrm{PH}$ and potentially the first effective oral therapy for inoperable group IV PH". However, Archer expresses disappointment that right heart function was not directly assessed in either trial. He also points out that, in patients with CTEPH, "the benefits with pulmonary endarterectomy ... surpass the benefits with riociguat". Therefore, "patients who are suitable candidates for surgery should continue to undergo surgery and not be relegated to an inferior treatment".

Hossein-Ardeschir Ghofrani, the lead investigator on both trials, counters Archers concerns by pointing out that all patients underwent baseline and follow-up right heart catheterization to measure right ventricular cardiac output. Furthermore, the CHEST-1 inclusion criteria were specifically designed to select "only inoperable patients or those who had already undergone surgery, but had remaining or recurrent $\mathrm{PH}$ ".

Patients from each trial were entered into extension studies (PATENT-2 and CHEST-2) to assess long-term outcomes. Furthermore, the investigators plan to follow up on preliminary trial data showing a possible benefit of riociguat in patients with either WHO group II or group III PH.

Gregory B. Lim 